\title{
Exactly solvable models in 2D semiclassical dilaton gravity and extremal black holes
}

\author{
O. B. Zaslavskii \\ Department of Mechanics and Mathematics, Kharkov V.N. Karazin's National \\ University, Svoboda
}

Sq.4, Kharkov 61077, Ukraine

E-mail:aptm@kharkov.ua

Previously known exactly solvable models of 2D semiclassical dilaton gravity admit, in the general case, only non-extreme black holes. It is shown that there exist exceptional degenerate cases, that can be obtained by some limiting transitions from the general exact solution, which include, in particular, extremal and ultraextremal black holes. We also analyze properties of extreme black holes without demanding exact solvability and show that for such solutions quantum backreaction forbids the existence of ultraextreme black holes. The conditions, under which divergencies of quantum stresses in a free falling frame can disappear, are found. We derive the closed equation with respect to the metric as a function of the dilaton field that enables one, choosing the form of the metric, to restore corresponding Lagrangian. It is demonstrated that exactly solvable models, found earlier, can be extended to include an electric charge only in two cases: either the dilaton-gravitation coupling is proportional to the potential term, or the latter vanishes. The second case leads to the effective potential with a negative amplitude and we analyze, how this fact affects the structure of spacetime. We also discuss the role of quantum backreaction in the relationship between extremal horizons and the branch of solutions with a constant dilaton.

PACS numbers: 04.60.Kz, 04.20.Jb, 04.70.Dy 


\section{INTRODUCTION}

Two-dimensional (2D) theories of dilaton gravity is a remarkable tool for better understanding the role of quantum effects in black hole physics in a more realistic four-dimensional case. The papers [1] gave a powerful incentive to detailed studying solutions within such theories and their properties (for recent review see, for example, [2], [3]). As long as physical processes are being investigated perturbatively, with a fixed classical metric chosen as the main approximation, solutions of field equations can be found directly. As regards selfconsistent solutions, they can be always found in the classical limit since, as is known, any classical 2D gravitation-dilaton is exactly integrable. (The issue of exact solutions becomes, however, non-trivial even classically if there is a scalar field coupled to matter [4], [5] (see [6] for the issue of normalizablity) or higher order terms in curvature are taken into account [7]. Yang-Mills and fermion fields can be also included into the scheme to give exact integrability [8], 9]. However, we will not consider such cases.) Meanwhile, physical demands (for instance, the necessity to trace the process of black hole evaporation with account for quantum backreaction that may become significant at its late stage) make it important to find exact solutions in the closed form for a self-consistent semiclassical (one-loop) problem. It turned out, however, that even in spite of relative simplicity of $2 \mathrm{D}$ theories, such exact solutions of semiclassical theory, in contrast to the classical one, may be found only for some special classes of Lagrangians [10] - 16].

The typical features of exact solutions found in these papers consist, in particular, in that corresponding black holes are nonextreme only and, moreover, the Hawking temperature is constant in the sense it does not depend on a horizon value of the dilaton. Apart from this, all exactly solvable models discussed in [10] - [16] refer to the uncharged case. However, the issue of extreme black holes, in connection with its obvious importance (for example, as potential candidates on the role of stable remnants after black hole evaporation), makes it actual to elucidate, whether it is possible to extend the approach to solvability from the uncharged case to the charged one to embrace the extreme case. Besides, even for uncharged 
black holes, it may happen that the solutions considered in [10] - 16 do not exhaust all possibilities and some other exact solutions for extreme black holes are also possible. We address these issues below. Meanwhile, the question of expanding classes of exactly solvable models in 2D dilaton gravity deserves treatment by itself, not only in the context of extreme black hole solutions. Thus, our aim is triple: (i) to expand families of exactly solvable models, (ii) take into account an electric charge, (iii) elucidate the conditions, under which extreme black holes exist in 2D semiclassical theories (in generic and exactly solvable models), and compare this situation with that in the classical theory.

The paper is organized as follows. In Sec. II we list field equations and the condition of exact solvability according to [16. In Sec. III we suggest alternative derivation of this condition, especially natural in the context of black hole solutions. In Sec. IV we consider the special class of solution with a constant dilaton field which does not fall into a general scheme. Sec. V is devoted to classical charged black holes where we generalize some known

examples. In Sec. VI it is shown that some non-trivial limiting transitions in models of [16] give rise to qualitatively new possibilities, including the existence of extreme black holes. In Sec. VII we relax the condition of exact solvability, analyze generic extreme black holes and examine the possibility of ultraextreme quantum-corrected black holes. Sec. VIII summarizes the main results.

\section{BASIC EQUATIONS}

Let us consider the system governed by the action

$$
I=I_{0}+I_{P L}, I_{0}=I_{g d}+I_{q}
$$

where gravitation-dilaton part

$$
I_{g d}=\frac{1}{2 \pi} \int_{M} d^{2} x \sqrt{-g}\left[F(\phi) R+V(\phi)(\nabla \phi)^{2}+U(\phi)\right]
$$

the contribution of the electromagnetic field is 


$$
I_{q}=-\frac{1}{4 \pi} \int_{M} d^{2} x \sqrt{-g} W(\phi) F_{\mu \nu} F^{\mu \nu}
$$

$F_{\mu \nu} F^{\mu \nu}=2 F_{01} F^{01} \equiv-2 E^{2}$ and we omit boundary terms that does not affect the form of field equations.

$I_{P L}$ is the Polyakov-Liouville action [17] incorporating effects of Hawking radiation and its backreaction on spacetime for a multiplet of $\mathrm{N}$ conformal scalar fields. It is convenient to write it down in the form [18], [19]

$$
I_{P L}=-\frac{\kappa}{2 \pi} \int_{M} d^{2} x \sqrt{-g}\left[\frac{(\nabla \psi)^{2}}{2}+\psi R\right]-\frac{\kappa}{\pi} \int_{\partial M} \psi k d s .
$$

The function $\psi$ obeys the equation

$$
\square \psi=R,
$$

where $\square=\nabla_{\mu} \nabla^{\mu}, \kappa=N / 24$ is the quantum coupling parameter. Varying the action with respect to the metric, we get

$$
T_{\mu \nu} \equiv T_{\mu \nu}^{(g d)}+T_{\mu \nu}^{(q)}+T_{\mu \nu}^{(P L)}=0
$$

where

$$
\begin{gathered}
T_{\mu \nu}^{(g d)}=\frac{1}{2 \pi}\left\{2\left(g_{\mu \nu} \square F-\nabla_{\mu} \nabla_{\nu} F\right)-U g_{\mu \nu}+2 V \nabla_{\mu} \phi \nabla_{\nu} \phi-g_{\mu \nu} V(\nabla \phi)^{2}\right\}, \\
T_{\mu \nu}^{(P L)}=-\frac{\kappa}{2 \pi}\left\{\partial_{\mu} \psi \partial_{\nu} \psi-2 \nabla_{\mu} \nabla_{\nu} \psi+g_{\mu \nu}\left[2 \square \psi-\frac{1}{2}(\nabla \psi)^{2}\right]\right\} \\
T_{\mu \nu}^{(q)}=-\frac{W}{2 \pi}\left(g_{\mu \nu} E^{2}+2 g^{\alpha \beta} F_{\mu \alpha} F_{\nu \beta}\right) .
\end{gathered}
$$

Variation of the action with respect to $\phi$ gives rise to the equation

$$
R F^{\prime}+U^{\prime}=2 V \square \phi+V^{\prime}(\nabla \phi)^{2}
$$

where prime denotes derivative with respect to $\phi$.

The Maxwell equations read 


$$
\left(W F^{\mu \nu}\right)_{; \nu}=0
$$

The electromagnetic field in our 2D case is antisymmetric:

$$
F_{\mu \nu}=-E e_{\mu \nu}
$$

where $e_{\mu \nu}=-e_{\mu \nu}$ and $e_{01}=(-g)^{1 / 2}$. Then it follows from (9) that

$$
T_{\mu}^{\nu(q)}=\frac{W E^{2}}{2 \pi} \delta_{\mu}^{\nu}
$$

Let us suppose that the auxiliary field $\psi$ depends on one variable $\phi$ only. Then the structure of field equations exhibits the existence of the Killing vector $\xi_{\alpha}=e_{\alpha}^{\beta} \mu_{; \beta}$, where $\mu^{\prime}=\tilde{F}^{\prime} \exp \left(-\int d \phi \frac{\tilde{V}}{\tilde{F}^{\prime}}\right)$

$$
\tilde{F}=F-\kappa \psi, \tilde{V}=V-\kappa \frac{\psi^{\prime 2}}{2} .
$$

It can be either spacelike or timelike. In what follows we restrict ourselves to the static case (timelike Killing vector). As in the static case both $\psi$ and $\phi$ depend on the spatial coordinate $x$ only $(\psi=\psi(x)$ and $\phi=\phi(x))$, we may exclude $x$ and express $\psi$ in terms of $\phi$ directly, so our assumption $\psi=\psi(\phi)$ is self-consistent and is valid for any static space-time.

Thus, we consider space-times which have no dynamics. Although, formally, radiative solutions do not fall into this class, the models under consideration are intimately related to them. To describe processes of formation and evaporation of black holes, we should add to gravitation-dilaton part of the action also classical matter fields, which in the simplest case represent shock waves. Then the systems we are dealing with in the present paper may be used to describe different parts of space-time before and after shock wave - an intial state, remnants that represent the result of evaporation, etc. (see, for instance, careful analysis of dynamic scenario of one particular exactly solvable model in [14]). Anyway, the description of static solutions and their properties (to which we restrict ourselves in the present article) is an essential ingredient for the full analysis of a more complex dynamic picture.

Let us return to the issue of field equations. Now the Gauss law takes the form $(\mu=0)$

$$
\left(W \sqrt{-g} F^{01}\right)_{, 1}=0,
$$


whence $F_{01}=-Q W^{-1} \sqrt{-g}, E^{2}=Q^{2} W^{-2}$, where the constant $Q$ has physical meaning of an electric charge. It follows from (9) that the dilaton equation reads

$$
R F^{\prime}+U^{\prime}=2 V \square \phi+V^{\prime}(\nabla \phi)^{2}-E^{2} W^{\prime},
$$

From the trace equations we obtain

$$
\begin{gathered}
U=\square \tilde{F}+W E^{2}, \\
A_{1} \square \phi+A_{2}(\nabla \phi)^{2}+E^{2}\left(\omega W+W^{\prime}\right)=0, \\
A_{1}=(u-\kappa \omega) \psi^{\prime}+\omega u-2 V, \\
A_{2}=(u-\kappa \omega) \psi^{\prime \prime}+\omega u^{\prime}-V^{\prime},
\end{gathered}
$$

where $u \equiv F^{\prime}, \omega \equiv \frac{U^{\prime}}{U}$.

It is worth noting that in the presence of an electric charge field equations retain the form, typical of the uncharged case, but with the redefinition $U \rightarrow U_{\text {eff }}$, where

$$
U_{e f f} \equiv U-Q^{2} W^{-1}
$$

If an electromagnetic field is absent $(E=0)$, the condition of exact solvability looks like [16]

$$
V=\omega\left(u-\frac{\kappa \omega}{2}\right)+C(u-\kappa \omega)^{2} .
$$

This condition was derived (with the absence of an electromagnetic field) in [16] from the demand that eq. (18) not contain gradient of the dilaton field, so that $A_{1}=A_{2}=0$. Meanwhile, before considering the situation with an electromagnetic field, it is instructive to look at the exact solvability in the case $E=0$ from another viewpoint. 


\section{NEW APPROACH TO EXACT SOLVABILITY}

Let us write down the metric in the Schwarzschild gauge:

$$
d s^{2}=-f d t^{2}+f^{-1} d x^{2}
$$

It is worth noting that field equations of the semiclassical system under discussion look very much like those for a pure classical, but with coefficients, shifted according to (14).

Then field equations take the following explicit form:

$$
\begin{gathered}
2 f \frac{\partial^{2} \tilde{F}}{\partial x^{2}}+\frac{\partial f}{\partial x} \frac{\partial \tilde{F}}{\partial x}-U-\tilde{V} f\left(\frac{\partial \phi}{\partial x}\right)^{2}=0 \\
\frac{\partial f}{\partial x} \frac{\partial \tilde{F}}{\partial x}-U+\tilde{V} f\left(\frac{\partial \phi}{\partial x}\right)^{2}=0 .
\end{gathered}
$$

In our gauge (22) the integration of eq. (5) gives us

$$
\frac{\partial \psi}{\partial x}=\frac{A-f_{x}^{\prime}}{f} .
$$

Let $\frac{\partial \phi}{\partial x} \equiv z(\phi)$. It is also convenient to take the difference of (23), (24) to get

$$
\frac{\partial^{2} \tilde{F}}{\partial x^{2}}=\tilde{V}\left(\frac{\partial \phi}{\partial x}\right)^{2} .
$$

Then it follows that

$$
\begin{gathered}
f=\chi \exp (-\rho), \chi^{\prime}=\frac{U}{z^{2} \tilde{u}} e^{\rho}, \rho=\int d \phi \alpha, \alpha \equiv \frac{\tilde{V}}{\tilde{u}}, \tilde{u}=u-\kappa \psi^{\prime} . \\
\tilde{V}=z^{-1} \frac{\partial}{\partial \phi}(\tilde{u} z)=\tilde{u}^{\prime}+\tilde{u} \frac{z^{\prime}}{z} .
\end{gathered}
$$

Inverting (28), we get

$$
\begin{gathered}
z=z_{0} \tilde{u}^{-1} e^{\rho}, z_{0}=\text { const }, \\
\chi^{\prime}=\frac{U \tilde{u}}{z_{0}^{2}} \exp (-\rho),
\end{gathered}
$$




$$
f=\exp (-\rho) \int_{\phi_{h}}^{\phi} d \phi \chi^{\prime}
$$

the point $\phi_{h}$ corresponds to the event horizon of a black hole, whence

$$
x=\int d \phi z^{-1}=z_{0}^{-1} \int d \phi \tilde{u} \exp (-\rho)
$$

Multiplying (25) by $\frac{\partial x}{\partial \phi}$ and taking into account (27) - (31), we get

$$
\frac{\psi^{\prime}-\rho^{\prime}}{\tilde{u}} \int_{\phi_{h}}^{\phi} d \phi^{\prime} w\left(\phi^{\prime}\right)=\frac{w\left(\phi_{h}\right)}{\tilde{u}\left(\phi_{h}\right)}-\frac{w(\phi)}{\tilde{u}(\phi)}, w \equiv \chi^{\prime} .
$$

Here we assumed that $\psi$ is regular on the horizon, whence in eq. (25) the constant $A=$ $\left(\frac{\partial f}{\partial x}\right)_{x=x_{h}}=z_{0} \frac{w\left(\phi_{h}\right)}{\tilde{u}\left(\phi_{h}\right)}$.

By their very meaning, the action coefficients $F, U, V$ should not depend on $\phi_{h}$, which is the characteristics of a particular solution only. The same is true for the quantity $\psi(\phi)$ and, therefore, for $\tilde{u}(\phi)$ and $w(\phi)$ as well. Differentiating (32) with respect to $\phi_{h}$, we get

$$
\frac{\left(\psi^{\prime}-\rho^{\prime}\right)}{\tilde{u}} w\left(\phi_{h}\right)=-\frac{\partial}{\partial \phi_{h}} \frac{w\left(\phi_{h}\right)}{\tilde{u}\left(\phi_{h}\right)} .
$$

There are two independent variables $\phi$ and $\phi_{h}$ in this equation. Solving it by separation of variables, we find that

$$
\begin{aligned}
& \psi^{\prime}=\rho^{\prime}+b \tilde{u}, \\
& \frac{w}{\tilde{u}}=U_{0} e^{-b \tilde{F}},
\end{aligned}
$$

$b$ and $U_{0}$ are constants. Comparing (35) with (30) and remembering the definitions of $\rho$ and $\omega \equiv U^{\prime} / U$, we find

$$
\begin{aligned}
& \tilde{V}=\omega \tilde{u}+b \tilde{u}^{2} \\
& \psi^{\prime}=\omega+2 b \tilde{u} .
\end{aligned}
$$

Eq. (37) is equivalent to (21), if one identifies constants $b=\frac{C}{1-2 \kappa C} \equiv \tilde{C}$. Throughout the present paper we put for simplicity $C=0$, so for exactly solvable models

$$
V=\omega u-\frac{\kappa \omega^{2}}{2}
$$

Thus, we arrive at the conditions of exact solvability, derived in [16] in a quite different way. 


\section{SOLUTIONS WITH CONSTANT $\phi$}

In the preceding section it was tacitly assumed that the dilaton field is not constant identically. Otherwise, this special case is needed to be discussed separately. Let $\phi=\phi_{0}=$ const. In terms of the spherically-symmetrical reduction from $4 \mathrm{D}$ theory to $2 \mathrm{D}$ one, the dilaton is analog of the coefficient $r^{2}$ at the angular part of the $4 \mathrm{D}$ interval. Therefore, the solutions with a constant dilaton are analogs of $r^{2}=$ const solutions, which are nothing else than the Bertotti-Robinson (BR) spacetimes. In doing so, the constant dilaton value corresponds to the horizon area.

For the constant dilaton the expression for $T_{\mu \nu}$ simplify:

$$
\begin{gathered}
T_{\mu \nu}^{(g d)}=-\frac{U\left(\phi_{0}\right)}{2 \pi} \delta_{\mu}^{\nu}, \\
T_{\mu \nu}^{(P L)}=-\frac{\kappa R}{\pi} \delta_{\mu}^{\nu} .
\end{gathered}
$$

We obtain from field equations, including the dilaton one:

$$
\begin{gathered}
U_{\text {eff }}\left(\phi_{0}\right)+\kappa R=0, \\
R F^{\prime}\left(\phi_{0}\right)+U_{\text {eff }}^{\prime}\left(\phi_{0}\right)=0,
\end{gathered}
$$

where $U_{\text {eff }}$ is taken from eq. (20).

In particular, for the string-inspired dilaton gravity theory [20] with

$$
F=e^{\phi}=V, U=\lambda e^{\phi}, W=e^{\phi}, U_{e f f}=\lambda e^{\phi}-Q^{2} e^{-\phi}
$$

our previous results [21] can be obtained from (41), (42). It was shown that in the absence of an electromagnetic field, the solutions with a constant dilaton in exactly solvable models are possible only due to quantum effects [18], [16]. However, now they appear in the classical domain as well.

There are two qualitatively different possibilities. 
1) $R=0$ (flat spacetime). This restricts the parameters of a system to some particular values. For example, if

$$
U_{e f f}=\lambda e^{2 \phi}+\Lambda-Q^{2} e^{\phi},
$$

the solution is possible only if $Q^{4}=4 \lambda \Lambda \equiv Q_{1}^{4}$, i.e. for a singled value of charge. This means such a balance between dilaton and electric forces, that combined action of both these sources does not curve spacetime.

2) $R \neq 0$. Then there are three possible cases:

$$
d s^{2}=-d t^{2} \mu^{2}+d l^{2}
$$

where $\mu=\left(\right.$ i) $e^{-c l}$, (ii) $\frac{\sinh c l}{c}$ or (iii) $\frac{\sin c l}{c}$. In the cases (i), (ii) $R=-2 c^{2}<0$, in (iii), $R=2 c^{2}>0$.

For (43) in the case (iii) we need, according to eqs. (41), (42) to combine $U_{\text {eff }}^{\prime}\left(\phi_{0}\right)<0$ and $U_{e f f}\left(\phi_{0}\right)<0$. This is possible only in the essentially quantum case: $\kappa>e^{\phi_{0}}$ and for $\lambda<0$ [21]. However, for (44) this can be achieved even classically and with $\lambda>0$, if $Q>Q_{1}$.

In the classical case, if eq. (41) has more than one root, any two successive roots correspond to the curvatures of different signs (provided $F^{\prime}$ does not change the sign) since derivative $U_{\text {eff }}^{\prime}$ changes its sign. With quantum backreaction taken into account the constant dilaton values move in such a way that

$$
\operatorname{sign} U^{\prime}=\operatorname{sign} F^{\prime} \operatorname{sign} U
$$

It was stressed in [22] that the constant dilaton solution appears just at the values which correspond to degenerate horizons of the basic branch of solution $(\phi \neq c o n s t)$. This conclusion was reached for classical systems. Now we will show that account for backreaction retains this relationship. Indeed, consider merging horizons. Then near the horizon $f=$ $f_{0}\left(x-x_{h}\right)^{2}+\ldots$ To have finite quantum stresses on the horizon, we should put $A=0$ in (25) that is equivalent to $T=T_{H}=0$ [23]. Then near the degenerate horizon

$$
\frac{\partial \psi}{\partial x}=-2\left(x-x_{h}\right)^{-1}+\ldots
$$


With account for (14), (47), eqs. (23) and (24) give us on the horizon the same condition $U_{\text {eff }}\left(\phi_{h}\right)+\kappa R\left(\phi_{h}\right)=0, R\left(\phi_{h}\right)=-2 f_{0}$ for a basic branch (non-constant dilaton). This condition corresponds just to (41) for the identically constant dilaton. For any regular function $\phi(x)$ we have $\square \phi=f^{\prime} \phi^{\prime}+f \phi^{\prime \prime}=0$ on the degenerate horizon, whence we get from the dilaton equation (10) the condition (42). Thus, if a basic branch admits merging horizons, the intimate connection between both types of solutions persists in the quantum case too.

It is worth stressing, however, that this kind of correspondence between two branches of solutions can be broken in two cases. First, when two horizons merge they form a degenerate horizon (an extremal black hole); however, such extremal horizons may be absent in some classes of solutions. For example, all black holes pertaining to exactly solvable models considered in [16] are non-extreme and constant dilaton solutions correspond to singularities of the main branch [18], [16]. Second, even if the main branch (with a non-constant dilaton) does admit extremal horizon, there exist solutions of field equations with a regular extremal horizon but infinite quantum stresses, when $A \neq 0$ [33], [34] (see also Sec. VIII below). Then derivation of eq. (41) for corresponding quantities on an extremal horizon of the main branch loses its validity since the equality $A=0$ was used there in an essential way (see above). For instance, the effective potential for extreme solutions in [33] $U=0$ on the horizon but the curvature $R \neq 0$ there, so eq. (41) is not fulfilled for the main branch. Thus, quantum backreaction may give rise to such extremal horizon which have no counterparts in the constant dilaton solutions.

\section{CLASSICAL CHARGED BLACK HOLES}

In the classical limit, when $\kappa=0$, any 2D dilaton gravity model becomes integrable. This fact is known. However, as some models of charged 2D dilaton black holes play an especial physical role in the context of the string theory [20], [24], [25] and are under intensive study up to now [26], we dwell upon on this case separately. There is an elaborated approach for 
obtaining solutions, which is based on simplifying Lagrangian due to excluding the kinetic term [27]. Instead, we give direct expressions for solutions in the original conformal frame without additional manipulations.

As now tilted coefficients coincide with the original ones, eqs. (27)-(31) give the complete solution of the problem since an unknown function $\psi(\phi)$ does not appear in their right hand side. All relevant formulas contain now, instead of the potential $U$, the effective potential (20).

Consider several examples.

Example 1. $V=e^{\phi}, F=e^{\phi}, U=\lambda^{2} e^{\phi}+\Lambda, W=e^{\phi}, U_{\text {eff }}=\lambda e^{\phi}+\Lambda-Q^{2} e^{-\phi}$. Let us choose the constant $z_{0}=\lambda_{0}$. Then

$$
f=1-\frac{2 M}{\lambda} e^{-\phi}+\frac{Q^{2}}{\lambda^{2}} e^{-2 \phi}+\frac{\Lambda \phi}{\lambda^{2}}
$$

In the limit $\Lambda \rightarrow 0$ the result of [20] is reproduced, the limit $Q \rightarrow 0$ corresponds to [26].

The solution for charged configurations may contain, in particular, extreme black holes. By definitions, for such black holes the Hawking temperature $T_{H}=\frac{f^{\prime}\left(x_{W}\right)}{4 \pi}=0\left(x_{h}\right.$ corresponds here to the horizon, where $\left.f\left(x_{h}\right)=0\right)$. Let everywhere $u \neq 0$, the function $F(\phi)$ is monotonic. Then it follows from (27) - (31) that $U_{\text {eff }}\left(\phi_{h}\right)=0$. In principle, it may happen that in the point $\phi_{h}$ also $U_{\text {eff }}^{\prime}\left(\phi_{h}\right)=0$. Then we get an "ultraextreme" black holes for which near the horizon $f=\operatorname{const}\left(x-x_{h}\right)^{3}$ (by definition, a black hole is ultraextreme if near the horizon $f \backsim\left(x-x_{h}\right)^{n}$ with $\left.n>2\right)$. This can be achieved if, say, we add the term of the kind $B e^{-\phi}$ to $U$. Such solutions were impossible for [20], 26].

Example 2. $F=e^{-2 \phi}, V=4 e^{-2 \phi}, U=-2 \lambda^{2} \sinh 2 \phi$ [28]. Choosing the constant $z_{0}=\lambda$ to ensure $f \rightarrow 1$ at $x \rightarrow+\infty$, we get

$$
x=-\frac{2}{\lambda}\left(\phi-\phi_{0}\right), f=\left(1-e^{2 \phi-2 \phi_{W}}\right)\left(1-e^{2 \phi+2 \phi_{h}}\right),
$$

where $\phi_{h}$ is the horizon value. If one identifies $a_{+}=\exp \left(2 \phi_{0}-2 \phi_{h}\right), a_{-}=\exp \left(2 \phi_{0}+2 \phi_{h}\right)$, we get

$$
f=\left(1-a_{+} e^{-\lambda x}\right)\left(1-a_{-} e^{-\lambda x}\right)
$$


that reproduces eq. (7) of [28].

\section{EXACT SOLUTIONS FOR SEMICLASSICAL CHARGED BLACK HOLES}

Let us now consider charged solutions in semiclassical theories of dilaton gravity and try to generalize exactly solvable models to take into account an electromagnetic field. Our strategy consists of two steps. First, we find exactly solvable solutions for the effective potential $U_{\text {eff }}(20)$. As both $U_{\text {eff }}$ and $\omega_{\text {eff }}=U_{\text {eff }}^{\prime} / U_{\text {eff }}$ contain the charge $Q$, this charge will, in general, enter the expressions for the functions $u(\phi)$ and $V(\phi)$. This is undesirable feature since the charge is characteristic of a particular solutions and does not appear in the original Lagrangian. In this sense, different exactly solvable models would correspond, generally speaking, to different $Q$, each for one value of $Q$ only. In other words, if some model was exactly solvable for a fixed value of $Q$, small variation of $Q$ would destroy exact solvability.

To exclude this and find the models, exactly solvable for any $Q$, we need to make the second step and get rid of $Q$-dependence in the action coefficients.

It is clear from (21) that the only way to get rid of the $Q$-dependence in $V$ is to demand that $\omega_{\text {eff }}$ not contain $Q$. Using the definition of $\omega_{e f f}$, we have

$$
\omega_{e f f}=\frac{U^{\prime}+Q^{2} W^{-2} W^{\prime}}{U-Q^{2} W^{-1}}
$$

There are only two possibilities here to achieve our goal.

\section{A. Case 1)}

1) $W=W_{0} U^{-1}$, where $W_{0}=$ const. Then $U_{\text {eff }} \backsim U$ and $\omega_{\text {eff }}=\omega=U^{\prime} / U$. In terms of eq. (18) this means that the third term is identically zero. Then the analysis of [16] (where an electromagnetic field was not taken into account) applies directly. The only difference is that the presence of an electric charge affects the value of the amplitude of the effective potential. If we write the potential in the form 


$$
U=4 \lambda^{2} \exp \left(\int_{0}^{\phi} d \phi \omega\right)
$$

then replacement $U$ by $U_{\text {eff }}$ means the change

$$
\lambda \rightarrow \bar{\lambda}=\lambda \sqrt{1-\frac{Q^{2}}{W_{0}}}
$$

(cf. [29], where, however, the system was supposed to be a pure classical). Correspondingly, all formulas look very much like for the uncharged case [16], if the parameter $\lambda$ is replaced by $\bar{\lambda}$.

Much more interesting possibilities arise in the second case since they can lead to qualitative changes in the structure of spacetime of exactly solvable models which we now turn to.

\section{B. Case 2)}

Let $U=0, W \equiv e^{\int d \phi \omega}$ be arbitrary, $U_{e f f}=-Q^{2} W^{-1}$. Then

$$
\omega_{e f f}=-\frac{W^{\prime}}{W}
$$

Using the results of [16], one can write (up to the constant factor) the explicit expression for the solution in the form

$$
\begin{gathered}
f=a\left(\tilde{F}_{h}-\tilde{F}\right) W, \tilde{F}_{h} \equiv \tilde{F}\left(\phi_{h}\right), \\
\frac{\partial x}{\partial \phi}=B^{-1} \tilde{F}^{\prime} W
\end{gathered}
$$

where $a$ and $B$ are constants, related, according to (17), by the equation

$$
a B^{2}=Q^{2}
$$

As in the case (1), the value of charge affects not the coordinate dependence of the metric explicitly but only the normalization factors like $\lambda$ and $a$.

The Riemann curvature 


$$
R=\frac{Q^{2}}{\tilde{F}^{\prime} W} \frac{\partial}{\partial \phi}\left[\frac{\left(\tilde{F}-\tilde{F}_{h}\right) W^{\prime}}{\tilde{F}^{\prime} W}\right]
$$

The roots of the equation $\tilde{F}^{\prime}\left(\phi_{s}\right)=0$ correspond to timelike or spacelike singularities, provided $W\left(\phi_{s}\right)$ and $W^{\prime}\left(\phi_{s}\right)$ are finite and nonzero. If the function $W$ has a zero at $\phi=\phi_{1}$, this root corresponds to the singular horizon. In general, the behavior of $W(\phi)$ near the point $\phi_{s}$ may affect the character of singularity and even remove it, making spacetime everywhere regular.

According to [30], for exactly solvable semiclassical dilaton gravity theories solutions of field equations can be written as

$$
\tilde{F}^{(0)}=h(\sigma) \equiv C+D e^{-\sigma \delta}+\kappa \gamma\left(1-\frac{\gamma}{2 \delta}\right) \sigma,
$$

where $\sigma$ is a conformal coordinate, $\tilde{F}^{(0)}=F-\kappa \psi_{0}$,

$$
\begin{gathered}
d s^{2}=f\left(-d t^{2}+d \sigma^{2}\right), \\
f=e^{-\psi_{0}-\delta \sigma}, \\
\psi_{0}=\ln U_{e f f}+\text { const }, \\
\psi=\psi_{0}+\gamma \sigma,
\end{gathered}
$$

Now the potential $U$ [30] should be replaced, as is explained above, by $U_{\text {eff }}=-Q^{2} W^{-1}$. If we write down the potential in the form $U \equiv \Lambda \exp \left(\int d \phi \omega\right)$, it follows from the trace equation that for exactly solvable models ( [30]) the constants obey the equation

$$
D \delta^{2}=\Lambda
$$

For the case under discussion $\Lambda=-Q^{2}<0$.

Thus, in contrast to [16], now $D<0$. Shifting $\sigma$, one can achieve $D=-1$. Introducing dimensionless coordinate $y=\frac{Q \sigma}{2}$ and choosing $\delta=-Q$ (the factor 2 is introduced to retain succession with previous papers [16], [30]), we get 


$$
\tilde{F}^{(0)}=-e^{2 y}-B y+C \equiv h(y),
$$

$B \equiv-\kappa \frac{2 \gamma}{Q}\left(1+\frac{\gamma}{2 Q}\right)$.

It is shown in [30] that the expression for the metric can be written for exactly solvable models in the form

$$
f=a e^{2 y} W
$$

For black holes, the coefficient $B$ arises due to deviation of temperature $T$ from its Hawking value $T_{H}$ [31]. Demand $T=T_{H}$ leads to $B=0$, in which case one can easily see that (66) is equivalent to (55).

For definiteness, consider the dilaton-electromagnetic coupling of the form $W=e^{2 \phi}$. Then $U_{\text {eff }}=-Q^{2} e^{-2 \phi}$. We choose the quantity $\tilde{F}$ corresponding to the CN [32] model that generalizes the RST [10] and BPP [14] ones:

$$
\tilde{F}^{(0)}=\exp (-2 \phi)+2 \kappa d \phi
$$

The amplitude of the potential is negative but, as the quantity $V$ for exactly solvable models contain $\omega \equiv \dot{U}_{\text {eff }}^{\prime} / U_{\text {eff }}$, the sign of the amplitude is irrelevant, and $V$ is the same as in [32]:

$$
V=4 \exp (-2 \phi)+2(1-2 d) \kappa, U_{\text {eff }}=-Q^{2} e^{-2 \phi} .
$$

It is convenient to calculate the curvature in the conformal frame according to the formula (where we have taken into account that for the model under discussion $\omega=-2$ )

$$
R=-\frac{Q^{2}}{2} f^{-1} \frac{\partial^{2} \phi}{\partial y^{2}}
$$

The main difference as compared to previously considered exactly solvable models [16], [30], [31] is that now the coefficient at $e^{2 y}$ in $h(y)$ changed the sign. At $y \rightarrow-\infty$ (near the horizon) this has a negligible effect. However, it affects the behavior of $h(y)$, when $y$ grows. Consider several possible cases separately and comment mainly on features of the spacetime which arise due to the negative sign of $D$ in (59).

1) $d=0$. 
a) $B \geq 0$. Now we have a region $-\infty<y \leq y_{0}$ between a horizon and the point $y_{0}$ such that $h\left(y_{0}\right)=0$. If $h^{\prime}\left(y_{0}\right) \neq 0$, it corresponds to a singularity, if $h^{\prime}\left(y_{0}\right)=0$, we have there a semi-infinite throat.

b) $B<0$. There are two roots $h\left(y_{1}\right)=h\left(y_{2}\right)=0$, if $C>\frac{|B|}{2} \ln \frac{|B|}{2 e}$ (otherwise, there are no admissible solutions at all). Near each root we have a singularity.

2) $d>0$. Now $\tilde{F}(\phi)$ has a minimum at $\phi=\phi_{0}, \tilde{F}^{(0) \prime}\left(\phi_{0}\right)=0$, eq. (65) for each $y$ has two solutions.

a) $B \geq 0$. As $h \rightarrow-\infty$ at right infinity, $y$ cannot grow unbounded, $y<y_{0}$, where $\tilde{F}^{(0)}\left(\phi_{0}\right)=h\left(y_{0}\right)$. Near this point, expanding relevant quantities, we get that in general $\phi-\phi_{0} \backsim \sqrt{y_{0}-y}, R \backsim\left(y-y_{0}\right)^{-3 / 2}$ diverges.

b) If $B=-|B|<0$, the function $h(y)$ has a maximum $h_{m}$. Solutions exist if $h_{m}>\tilde{F}\left(\phi_{0}\right)$, in which case the admissible region is bounded by two singularities: $y_{1} \leq y \leq y_{2}$, where $\tilde{F}\left(\phi_{0}\right)=h\left(y_{1,2}\right)$.

Thus, in both cases a), b) the branch with the flat right infinity, typical of the case $D=1$, now disappears..

3) $d<0$. The function $\tilde{F}^{(0)}(\phi)$ is monotonic. Then, the branch with a linear dilaton vacuum $\phi=-y$ at right infinity, typical of the case $D=1$, does not longer exists. Now at $y \rightarrow \infty$ the dilaton $\phi=|d|^{-1} \kappa^{-1} e^{2 y}$. The proper distance $l \backsim \int d y \sqrt{f}$ diverges in this limit, eq. (69) shows that the curvature $R \backsim \exp \left(-\frac{2}{|d| \kappa} e^{2 y}\right)$ is bounded. Thus, we have now a semi-infinite throat at $y \rightarrow \infty$ that has no counterpart in the case $D=1$.

\section{DEGENERATE MODELS AND EXTREMAL HORIZONS}

The general form of solutions (65) implies that the parameters $\delta, \gamma, D$ entering (59) are finite and nonzero. Meanwhile, there exist some special (degenerate) cases, when the parameter $\delta$ vanishes. Then the form of the function $h$ in the right hand side of (65) changes qualitatively. Below we discuss the corresponding cases separately.. It is especially interesting that they contain, in particular, extremal black holes $\left(T_{H}=0\right)$. For comparison, 
let me recall that in generic exactly solvable models considered in [16], [30 such solutions were absent. Extremal horizons with $\delta \neq 0$ were obtained in 33 by expense of relaxing the finiteness of quantum stresses on the horizon that needs imposing some severe restrictions on the form of the coefficient $\tilde{F}$ near the horizon. Meanwhile, now (for degenerate models) extremal horizons can be obtained, as will be seen, in a more natural way.

\section{A. $\delta \rightarrow 0, \gamma \rightarrow 0, D$ is finite}

Let not only $\delta \rightarrow 0$, but also $\gamma \rightarrow 0$ with $D$ remaining finite, so the potential $U_{\text {eff }}=0=$ $\bar{\lambda}$. It can be achieved at $Q=0, U=0$ or at $Q^{2}=W_{0}$, see (53). Then eq. (59) has a non-trivial limiting transition, if $\delta \sim \gamma^{2}: \tilde{F}=a \sigma+C+D, a \equiv-\frac{\kappa \gamma^{2}}{2 \delta}$. After an obvious rescaling we have

$$
\tilde{F}=\sigma
$$

where $f$ can be found from (72) and (74).

It is also instructive to see, how the solutions of this type can be obtained in the Schwarzschild gauge (22) which was used in 33] to realize, why the models under discussion do not fall into the classes discussed in [16. It follows from the integration of eq. (5) in the Schwarzschild gauge (22) that

$$
f=e^{-\psi} \chi, \chi=A \int_{\phi_{h}}^{\phi} d \phi^{\prime} \tilde{F}^{\prime}\left(\phi^{\prime}\right) e^{-\psi}+A_{0}
$$

It was assumed in [16] that $A \neq 0, A_{0}=0$ that corresponds to the horizon at $\phi=\phi_{h}$, when $\chi=f=0, \psi$ being finite on the horizon. Meanwhile, another type of black hole solutions with a regular horizon is also possible, if $A=0, A_{0} \neq 0, \psi \rightarrow \infty$ on the horizon. Then

$$
\begin{gathered}
f=f_{0} e^{-\psi}, \psi=\int d \phi \omega_{e f f} \\
x=x_{0} \mu, \mu^{\prime}=\tilde{F}^{\prime} e^{-\psi}
\end{gathered}
$$


As now $U_{\text {eff }}=0$, the definition $\omega_{\text {eff }}=U_{\text {eff }}^{\prime} / U_{\text {eff }}$ loses its sense but the formula (21) for the condition of exact solvability is still valid. It changes its meaning: due to the condition $U_{\text {eff }}=0$ we may impose the dependences $V(\phi)$ and $u(\phi)$ at our will, find $\omega$ from (21) and obtain $\psi^{\prime}$ from (72):

$$
\psi_{ \pm}^{\prime}=\frac{u}{\kappa} \pm \sqrt{\left(\frac{u}{\kappa}\right)^{2}-\frac{2 V}{\kappa}} .
$$

If we want to have a well-defined classical limit $\kappa \rightarrow 0$, we should take $\psi^{\prime}=\psi_{-}^{\prime}=\omega_{\text {eff }}$.

Taking into account that the Schwarzschild and conformal gauge are related according to $d x=d \sigma f$, one can establish easily the equivalence of formulas (70) and (72) - (74).

Consider explicit examples. Let $\omega_{e f f}=$ const $\equiv \omega_{1}>0, \tilde{F}=e^{\omega_{2} \phi}+\alpha \phi, V=\omega_{1} \omega_{2} e^{\omega_{2} \phi}+$ $\alpha \omega_{1}+\kappa \frac{\omega_{1}^{2}}{2}, \omega_{2}$ is a constant. Then $f=f_{0} e^{-\omega_{1} \phi}, x=x_{1} \exp \left[\left(\omega_{2}-\omega_{1}\right) \phi\right]+x_{2} e^{-\omega_{1} \phi}+x_{h}$, where $x_{1}, x_{2}, f_{0}$ are another constants, the quantity $\psi=\omega_{1} \phi$. Near the horizon $\phi \rightarrow \infty$ and $\psi$ diverges in agreement with the statement made above. If $\omega_{2}=\omega_{1} / 2, f \backsim\left(x-x_{h}\right)^{2}$ and we get the extreme black hole. At infinity we have $f \backsim x, R \rightarrow 0$, thus this extreme black hole is accelerated.

If $\omega_{2}=\omega_{1} \frac{(n-1)}{n}, f \backsim\left(x-x_{h}\right)^{n}$. Thus, for $n>2$ the horizon is "ultraextreme" in the sense that not only $f\left(x_{h}\right)=0$, but also $f^{\prime}\left(x_{h}\right)=0$.

If $\omega_{2}<0$, we have near the horizon $f \backsim\left(x-x_{h}\right)$, so the black hole is nonextreme. In particular, if $\omega_{2}=-\omega_{1}$, the expansion has the form $f=a_{1}\left(x-x_{h}\right)+a_{2}\left(x-x_{h}\right)^{2}$, so the horizon is regular, $R\left(x_{h}\right)=-2 a_{2}$.

Thus, depending on the properties of $\omega(\phi), F(\phi)$ and $V(\phi)$, we have a diversity of possibilities, including extreme black holes $\left(T_{H}=0\right)$. This is in sharp contrast with exactly solvable models in [16], where the Hawking temperature $T_{H}=$ const $\neq 0$.

$$
\text { B. } \delta \rightarrow 0, D \rightarrow-\infty, \gamma \neq 0 \text {. }
$$

Let $C=C^{\prime}-D$, where $C^{\prime}$ is finite,

$$
D=D_{0}+D_{1} \delta^{-1}+D_{2} \delta^{-2}
$$


where $D_{0}, D_{1}$ and $D_{2}$ are some constants. Eq. (59) has a well defined limit $\delta \rightarrow 0$, provided the coefficient $D_{2}$ is chosen to kill the divergent terms: $D_{2}=-\frac{\kappa \gamma^{2}}{2}$. Then, getting rid of the terms, linear in $\sigma$, by a simple shift, and, for definiteness, taking the constant term positive, we obtain

$$
\tilde{F}^{(0)}=\kappa \frac{\gamma^{2}}{4}\left(\sigma_{0}^{2}-\sigma^{2}\right)
$$

$\sigma_{0}^{2}=$ const. It is seen that the existence of the degenerate case is due to quantum effects only $(\kappa \neq 0)$.

From the trace equation

$$
U_{e f f}=-\frac{\kappa \gamma^{2}}{2 f}, f \backsim W
$$

Consider an example.

$$
W=e^{2 \phi}, F=e^{2 \phi}-\alpha e^{-2 \phi}+\beta \phi, \tilde{F}^{(0)}=e^{2 \phi}-\alpha e^{-2 \phi}+\tilde{\beta} \phi, \tilde{\beta}=\beta+2 \kappa, \omega=-2, \alpha>0 .
$$

Then

$$
f \backsim e^{2 \phi}
$$

There are two horizons at $\sigma= \pm \infty, \phi \rightarrow-\infty$, near which

$$
f \backsim \sigma^{-2} \backsim\left(x-x_{h}\right)^{2},
$$

where $x$ is the Schwarzschild coordinate. Thus, the solution represent an extreme black holes with two symmetric horizons. It is also clear that in the case $\tilde{\beta}=0$ the fractional correction to $f$ have the order $\sigma^{-2}$, so near the horizon $f=A_{1}\left(x-x_{h}\right)^{2}+A_{2}\left(x-x_{h}\right)^{4}$. It is known that the energy measured by a free falling observer is proportional to $\frac{f^{\prime \prime \prime}}{f^{\prime}}$ [35, 223. Thus, this quantity is finite and there are no thermal divergencies on the event horizon, in contrast to a generic case.

In another case, if $\tilde{F}$ in $(78)$ contains the term $c e^{-3 \phi}$, with $c<0$, near the horizon we have $f \backsim \sigma^{-4 / 3} \backsim\left(x-x_{h}\right)^{4}$. Thus, a black hole is "ultraextreme". 


\section{GENERIC EXTREMAL SEMICLASSICAL BLACK HOLES}

In the preceding sections we mainly considered exactly solvable models and found that only in some special limits one can hope to find for such models solutions of the extremal black hole type. Thus, extremality and exact solvability rather seldom come into play simultaneously. Meanwhile, the issue of extremal black holes is important by itself and, therefore, it is of interest to elucidate, what information about corresponding solutions can be extracted from basic equation without severe restriction to exactly solvable models only.

In what follows we are interested in such extremal black holes that quantum stresses remain bounded on a horizon. In principle, regular extremal horizons may exist even if this restriction is relaxed [33], [34] but we do not discuss here these rather special cases and assume that, as usual, a static extreme black hole with a regular horizon realizes the Hartle-Hawking state with the temperature $T=T_{H}=0$.

It is seen from eqs. (8) and (25) that the expression for $T_{1}^{1(P L)}$ can be rewritten, as

$$
T_{1}^{1(P L)}=-\frac{\kappa}{4 \pi f}\left[A^{2}-f_{x}^{\prime 2}\right]
$$

As for extremal horizons $f_{x}^{\prime}\left(x_{h}\right)=0$, the condition of regularity demands that $A=0$. Then, according to (25),

$$
f=f_{0} e^{-\psi}
$$

where $f_{0}$ is a constant. (Recall that in [16] we assumed that $\psi$ was regular on the horizon and took the quantity $A=f^{\prime}\left(x_{h}\right) \neq 0$ in agreement with the non-extreme character of black holes considered in [16].)

One can rewrite (24) and (26) as

$$
\begin{gathered}
\left(V-\psi^{\prime} u+\kappa \frac{\psi^{\prime 2}}{2}\right) z^{2}=U_{e f f} e^{\psi} \\
C_{1} z+C_{2} \frac{\partial z}{\partial \phi}=0
\end{gathered}
$$




$$
C_{1}=u^{\prime}-V+\kappa\left(\frac{\psi^{\prime 2}}{2}-\psi^{\prime \prime}\right), C_{2}=\left(u-\kappa \psi^{\prime}\right)
$$

(recall that $\left.z \equiv \frac{\partial \phi}{\partial x}\right)$.

For exactly solvable models, when eq.(38) is satisfied, the left hand side of eq. (83) vanishes and we return to the degenerate case $U_{\text {eff }}=0$, considered above - see eqs. (71) - (74). From now on, we assume that $U_{\text {eff }} \neq 0$. This means that the condition (38) is abandoned.

Let $z \equiv \sqrt{Y(\phi)}, Y \equiv \frac{a}{b}$. We want to derive from (83), (84) the closed equation for the single quantity $\psi$. We have

$$
2 C_{1}+C_{2}\left(\frac{a^{\prime}}{a}-\frac{b^{\prime}}{b}\right)=0
$$

Here $a=U_{\text {eff }} e^{\psi}, b=V-\psi^{\prime} u+\kappa \frac{\psi^{\prime 2}}{2}$ (henceforth we write down for shortness $U$ instead of $\left.U_{\text {eff }}\right)$. After some manipulations we get the equation $\left(y \equiv \psi^{\prime}\right)$

$$
\begin{gathered}
y^{\prime} d+d_{3} y^{3}+d_{2} y^{2}+d_{1} y+d_{0}=0, \\
d=\left(u^{2}-2 V \kappa\right), \\
d_{3}=\frac{\kappa}{2}(u-\kappa \omega) \\
d_{2}=-u^{2}+\left(\frac{3 \omega u}{2}-V\right) \kappa \\
d_{1}=u\left(3 V-\omega u-u^{\prime}\right)+\kappa\left(V^{\prime}-\omega V\right) \\
d_{0}=V\left(2 u^{\prime}-2 V+\omega u\right)-u V^{\prime}
\end{gathered}
$$

The classical case corresponds to $\kappa=0$. Then

$d=u^{2}, d_{3}=0, d_{2}=-u^{2}, d_{1}=u\left(3 V-\omega u-u^{\prime}\right)$. Let $V \equiv u \alpha$. Then eq. (87) turns into

$$
y^{\prime}-y^{2}+y\left(3 \alpha-\omega-\frac{u^{\prime}}{u}\right)+\alpha\left(\frac{u^{\prime}}{u}-2 \alpha+\omega\right)-\alpha^{\prime}=0
$$


By substitution $y=\eta+\alpha$ we get

$$
\begin{gathered}
\eta^{\prime}-\eta^{2}+X \eta=0, X \equiv\left(\alpha-\omega-\frac{u^{\prime}}{u}\right) . \\
\eta \equiv-\frac{\chi^{\prime}}{\chi} . \\
\chi^{\prime \prime}+\chi^{\prime} X=0 . \\
\chi^{\prime}=\exp \left[-\int d \phi Y(\phi)\right] .
\end{gathered}
$$

This coincides with eqs. (27). - (31), in which $\kappa=0$ is put in the right hand sides.

Consider now the quantum case. Following the procedure of [27], we can exclude the kinetic term and take the coefficient $F$ as a new dilaton field, provided $F(\phi)$ is monotonic: $V=0, F(\phi) \Longrightarrow \phi$. Then

$$
d=1, d_{3}=\frac{\kappa(1-\kappa \omega)}{2}, d_{2}=-1+\frac{3 \omega \kappa}{2}, d_{1}=-\omega, d_{0}=0
$$

We get

$$
y^{\prime}+\frac{\kappa(1-\kappa \omega)}{2} y^{3}+\left(-1+\frac{3 \omega \kappa}{2}\right) y^{2}-\omega y=0 .
$$

If we want to have $y=-\frac{2}{\phi}$ (zero temperature Bertotti-Robinson spacetime), we obtain [22], [36]

$$
\omega=\frac{1}{\phi+\kappa}, U=U_{0}(\phi+\kappa)
$$

In principle, we may find $\omega$ in terms of $y$ and $y^{\prime}$ from (99) and adjust $\omega$ and $U$ to have any desired behavior $y(\phi)$, typical of an extremal black hole. This means that equality $y=-2 / \phi$ should be fulfilled near the horizon only, so the curvature does not need to be constant everywhere. In turn, it means that, on the basis of eq. (87), one can describe diversity of models with desired features. To some extent, the procedure under discussion (adjusting the potential to fixed solutions or properties) is similar to finding the stress-energy 
tensor $T_{\mu \nu}$ from geometry of spacetime, using Einstein equations. In general relativity, such a procedure is not very natural since it leaves the question about physical nature of $T_{\mu \nu}$ unresolved. However, for 2D models it looks more reasonable since we simply fixed the form of the potential. Apart from this, in this approach one is able to describe geometries of physical interest, including, for-example, the potential (100), relevant for self-consistent scenario of black-hole evaporation [36].

\section{A. Extremal horizons with and without divergencies of quantum stresses}

As is known, in the extreme case the coordinate dependence of a dilaton near the horizon in the Schwarzschild gauge is non-analytical, that is connected with the appearance of divergencies of the quantum stresses on the horizon in the reference frame of a free falling observer. This was shown in 35] for a particular 2D model, obtained by spherically-symmetrical reduction from 4D Lagrangian. It is now instructive to trace, on the basis of eq. (87), how these divergencies appear in the general case. Let a horizon be situated at $\phi=0$, where $y \rightarrow \infty$ according to (82). Dividing eq. (87) by $y^{3}$ and taking the limit $y \rightarrow \infty$, we find that if we want this limit indeed to correspond to the horizon, $d_{3} \rightarrow 0, \omega \rightarrow u / \kappa$ on the horizon. Expanding $d_{3}$ in terms of $\phi$ and substituting the asymptotic expression

$$
y=-\frac{\varepsilon}{\phi}, \varepsilon \equiv \frac{2}{1+\tau}
$$

into (87), we get, equating terms at the $\phi^{-2}$ :

$$
\begin{gathered}
\tau^{2}+3 \tau-2 \kappa \nu=0, \\
\nu \equiv \lim _{\phi \rightarrow 0}\left(\frac{2 V+u^{\prime}-\frac{U^{\prime \prime}}{U^{\prime}} \kappa}{u^{2}-2 V \kappa}\right) .
\end{gathered}
$$

Eqs. (82), (101) give us the asymptotic form $f=\phi^{\varepsilon}$ near the horizon. Comparing it with the coordinate asymptotics $f=\operatorname{const}\left(x-x_{h}\right)^{2}$, typical of an extremal horizon, we get $\phi \backsim\left(x-x_{h}\right)^{1+\tau}$. In the classical limit $\kappa \rightarrow 0 \varepsilon=2$ and dilaton dependence of the metric $f=\operatorname{const} \phi^{2}$ becomes analytical: $f=\operatorname{const} \phi^{2}$. 
In the model considered in 35] $F=e^{-2 \phi}, u=-2 e^{-2 \phi}, U=2-2 Q^{2} e^{2 \phi}, V=2 e^{-2 \phi}$, $e^{-2 \phi_{h}} \equiv d_{h}$. Then (103) gives us

$$
\nu=\frac{3}{d_{h}-\kappa}
$$

in full accord with [35].

If $\nu=0$, we get $\tau=0$ - divergencies under discussion do not appear on the horizon (the root $\tau=-3$ is not suitable since we must have $\varepsilon>0$ to get the horizon at $\phi=0)$. For example, this takes place for the model $F=e^{-\phi}, V=2 e^{-\phi}, U=U_{1}+U_{2} e^{-5 \phi}$.

\section{B. Ultraextreme black holes}

It was noticed in Sec. $\mathrm{V}$ that $2 \mathrm{D}$ dilaton gravity admits such a kind of black holes that near the horizon

$$
f \backsim\left(x-x_{h}\right)^{n}, n>2
$$

("ultraextreme" black holes). Now we will show that account for quantum backreaction changes this result drastically.

It follows from (84) that

$$
\frac{\partial \ln z}{\partial \phi}=\frac{\kappa\left(\frac{y^{2}}{2}-y^{\prime}\right)+u^{\prime}-V}{\kappa y-V},
$$

whence in the limit $y \rightarrow \infty$, corresponding to the horizon, we obtain

$$
\frac{\partial \ln z}{\partial \phi}=\frac{y}{2}-\frac{y^{\prime}}{y}+\ldots
$$

On the other hand, comparing the asymptotic behavior $f \backsim\left(x-x_{h}\right)^{n}$ to the general formula (82), we find that near the horizon

$$
\frac{\partial \ln z}{\partial \phi}=\frac{y}{n}-\frac{y^{\prime}}{y}+\ldots
$$

Comparing (107) and (108), we see that $n=2$. Thus, ultraextreme black holes are impossible if quantum effects are taken into account. 
It is instructive to trace the origin of discrepancy between the classical and quantum cases. If the quantum-coupling parameter $\kappa=0$ from the very beginning, it is seen from

(106) that $\frac{\partial \ln z}{\partial \phi}$ remains bounded on the horizon, so do $z$ and $z^{\prime}$. Then one can infer from (83) (where the terms with $\kappa$ are dropped) and (82) that for a black hole of the type (105) the potential behaves near the horizon like $U \backsim f^{\prime} \backsim\left(x-x_{h}\right)^{n-1} \backsim\left(\phi-\phi_{h}\right)^{n-1}$. In turn, this means that $\omega=(n-1) / \phi$. Then one can check that the solution under discussion obeys eq. (93), where $y=-n / \phi$ near the horizon.

On the other hand, it is just terms with $\kappa$ (which are absent for a classical system) which dominate both the numerator and denominator in (106) and in eq. (83) since they contain $y$ and $y^{\prime}$ and this is just the reason why the structure of solutions changes qualitatively.

It is worth noting that in general relativity the issue of influence of quantum backreaction on the character of solutions is highly non-trivial, because of absence of exact solutions and mathematical complexities. It was shown in [37], 38] that in general relativity the presence of quantum backreaction is compatible with the existence of extremal black holes. The similar conclusion was reached in [39] for 2D theories with non-conformal scalar fields. However, as we saw above, the situation looks differently for ultraextreme solutions: quantum effects forbid them.

The statement about impossibility of ultraextreme semiclassical black hole refer to the generic case $U \neq 0$. However, if $U=0$, such solutions do become possible as we saw in some examples in Sec. VI following eq. (74).

\section{SUMMARY}

The condition of exact solvability in 2D dilaton semiclassical gravity (38) involves, via the effective potential, the value of an electric charge. This makes it, in general, impossible to generalize these conditions to have exact solutions for any value of charge. This is in sharp contrast, say, with general relativity where the Reissner-Nordstöm solution is known for an arbitrary charge. It turned out that there are only two ways to circumvent the obstacle 
under discussion and exclude the charge from $U_{\text {eff }}$ that leads to two possibilities. In the first case the solutions look very much like uncharged ones but with new parameters which contain the electric charge. In the second case the original potential $U=0$, and $U_{\text {eff }}$ is due to the dilaton-Maxwell coupling entirely. Again, the structure of exact solutions follows from general approach developed for the uncharged case [16 but now the amplitude of the effective potential is negative. First, it affects the structure of spacetime, as compared to the case $U_{\text {eff }}>0$, typical of uncharged solutions. Second, it admits the special "degenerate" class of exactly solvable models which is absent in the case of potentials with a positive amplitude.

The relationship between the generic exactly solvable case and its special degenerate subclass is of interest also on its own, independent of the presence of an electric charge. We described this relationships in terms of some universal function $q(\sigma)$, where $\sigma$ is a conformal coordinate. Some non-trivial limiting transitions change its character completely and show that there exist exact solutions qualitatively different from those described in [16]. The main new feature here is that these exact solutions may include extreme black holes.

On the other hand, usual (non-degenerate) exactly solvable models admit only nonextreme black holes. Therefore, the attempt to find extreme black holes beyond the degenerate subfamilies of exactly solvable models forced us to relax the condition (38) of exact solvability at all and consider generic extreme black holes $\left(U_{\text {eff }} \neq 0\right)$ in the Hartle-Hawking state.

It is instructive to summarize in a table, in which cases $2 \mathrm{D}$ semiclassical dilaton theories admit extreme black holes.

\begin{tabular}{|l|l|l|l|}
\hline & $\delta=0 ; \gamma, A, U \neq 0$ & $\delta, \gamma, A, U=0$ & $A=0, U \neq 0$ \\
\hline Exactly solvable & degenerate subclass & degenerate subclass & - \\
\hline$q(\sigma)$ & $\frac{\kappa \gamma^{2}}{4}\left(\sigma_{0}^{2}-\sigma^{2}\right)$ & $\sigma$ & - \\
\hline EBH & admissible & admissible & mandatory \\
\hline Divergencies & + & - & - \\
\hline UEBH & admissible & admissible & forbidden \\
\hline
\end{tabular}


Here EBH and UEBH mean extreme and ultraextreme black holes. In the last but one row we indicated, whether or not quantum stresses on a horizon diverge (that is determined by the coefficient $A$ in (81). To avoid possible confusion, we want to pay attention that information in the table concerns divergencies in the original (static) reference frame, whereas those found in 355 refer to a free falling observer. The fact, that divergencies of the first kind are compatible with finiteness of curvature, gives us one more example (apart from those in [33], 34] ) that in dilaton gravity infinite backreaction may be compatible with a regular horizon.

We have managed to derive one closed equation with respect to the auxiliary field $\psi(\phi)$ which governs the behavior of the system. In particular, with the help of this equation we analyzed in a general setting divergencies of quantum stresses on the horizon [35], [23] and found the conditions when these divergencies completely cancel.

In a sense, it is rather hard to combine exact solvability with extremal horizons: only degenerate cases of the general scheme [16] admit such a combination. However, information which can be extracted from the equation for $\psi(\phi)$, enables us to find exact solutions in another, more restricted sense: choosing any desirable behavior of the metric as a function of dilaton, we restore the Lagrangians which ensure such a behavior ("inverse problem").

We also found and analyzed exact solutions without the condition of exact solvability for a special branch of solutions with the constant dilaton field. It is shown that the correspondence between these solutions and extremal horizon of the main branch remains intact in spite of the presence of backreaction, but with the reservation that quantum stresses remain bounded on the horizon. If the later condition is relaxed, there exist regular extremal horizons having no counterparts among solutions with a constant dilaton field.

As far as the role of quantum backreaction is concerned, it turns out also that, although it does not affect the very existence of extremal black holes, it forbids their ultraextreme versions (except some special cases). This stimulates to examine this issue further for a more realistic 4D case, where such solutions are known on the classical level - for instance, among Reissner-Nordström-de Sitter solutions. 
[1] C.G. Callan, S. Giddings, J.A. Harvey J. A and A. Strominger, Phys. Rev. D 45 (1992) R1005;

T. Banks, A. Dabholkar, M. R. Douglas, and M. O. 'Loughlin, Phys. Rev. D 45 (1992 ) 3607.

[2] S.Nojiri and S. Odintsov, Int. J. Mod. Phys. A 16 (2001 ) 1015.

[3] D. Grumiller, W. Kummer and D. V. Vasilevich, Phys.Rept. 369 (2002) 327.

[4] A. T. Fillipov and V. G. Ivanov, Phys.Atom.Nucl. 61 (1998) 1639 (hep-th/9803059).

[5] A. T. Fillipov, Mod.Phys.Lett. A11 (1996) 1691.

[6] E. Elizalde and S.D. Odintsov, Nucl. Phys. B399 (1993) 581.

[7] E. Elizalde, P. Fosalba-Vela, S. Naftulin, S. D. Odintsov, Phys. Lett. B 352 (1995) 235.

[8] T. Kloesch and T. Strobl, Class. Class.Quant.Grav. 13 (1996) 965-984; Erratum-ibid. 14 (1997) 825.

[9] H Pelzer and T. Strobl, Class.Quant.Grav. 15 (1998) 3803.

[10] J. G. Russo, L. Susskind, and L. Thorlacius, Phys. Rev. D 46 (1992) 3444; Phys. Rev. D 47 (1992) 533 .

[11] A. Bilal and C. G. Callan, Nucl. Phys. B 394, 73 (1993).

[12] S. P. de Alwis, Phys. Rev. D 46 (1992) 5429.

[13] G. Michaud and R. C. Myers, Two-Dimensional Dilaton Black Holes, gr-qc/9508063.

[14] S. Bose, L. Parker and Y. Peleg, Phys. Rev. D 52 (1995) 3512.

[15] Y. Kazama, Y. Satoh, and A. Tsuichiya, Phys. Rev. D 51 (1995) 4265.

[16] O. B. Zaslavskii, Phys. Rev. D 59 (1999) 084013.

[17] A. M. Polyakov, Phys. Lett. B 103 (1981) 207. 
[18] S. N. Solodukhin, Phys. Rev. D 53 (1996) 824.

[19] V. P. Frolov, W. Israel, and S. N. Solodukhin, Phys. Rev. D 54 (1996) 2732.

[20] V. P. Frolov, Phys. Rev. D 46 (1992) 5383.

[21] O. B. Zaslavskii, Phys. Lett. B 424 (1998) 271.

[22] J. Cruz, A. Fabbri, D. J. Navarro and J. Navarro-Salas, Phys. Rev. D 61 (1999) 024011.

[23] D.J. Loranz, W.A. Hiscock and P.R. Anderson, Phys. Rev. D 52 (1995) 4554.

[24] G.W.Gibbons and M.J.Perry, Int.J.Mod.Phys. D 1 (1992) 335.

[25] C. R. Nappi and A. Pasquinucci, Mod. Phys. Lett. A 7 (1992) 3337.

[26] N. Berkovits, S. Gukov and B.C. Vallilo Nucl.Phys. B 614 (2001) 195.

[27] J. Gegenberg, G. Kunstatter, D. Louis-Martinez, Phys. Rev. D 51 (1995) 1781.

[28] S. Monni and M. Cadoni, Class. Quant. Grav. 14, (1997) 517.

[29] Y. Kiem, C.-Y. Lee and D. Park, Class. Quant. Grav. 15 (1998) 2973.

[30] O.B. Zaslavskii, Phys. Lett. B 459 (1999) 105.

[31] O.B. Zaslavskii, Phys. Rev. D 61 (2000) 064002.

[32] J. Cruz and J. Navarro-Salas, Phys. Lett. B 375 (1996) 47.

[33] O.B. Zaslavskii, Phys. Lett. B 475 (1999 ) 33.

[34] O. B. Zaslavskii, Entropy of semiclassical 2D dilaton black holes away from the Hawking temperature, hep-th/0208206 (To be published in Mod. Phys. Lett. A).

[35] S. Trivedi, Phys. Rev. D 47 (1993) 4233.

[36] A. Fabbri, D. J. Navarro and J. Navarro-Salas, Phys. Rev. Lett. 85 (2000) 2434.

[37] D. A. Lowe, Phys. Rev. Lett. 87 (2001) 029001. 
[38] J. Matyjasek and O. B. Zaslavskii, Phys. Rev. D 64 (2001) 104018.

[39] C. Barbachoux and A. Fabbri, Phys. Rev. D 66 (2002) 024012. 